\title{
A Case of Acral Lick Dermatitis in a Dog with Multiple Lesions
}

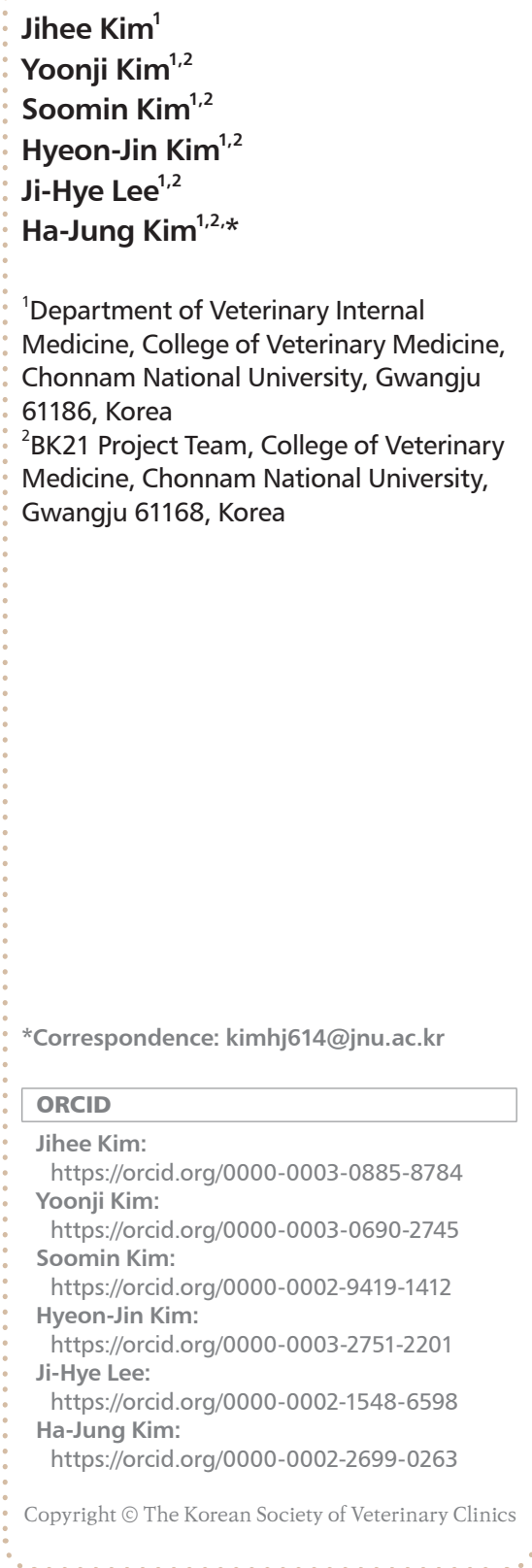

Abstract A 7-year-old intact male Maltese dog was presented with firm, raised, erythematous, alopecic, and pruritic skin lesions on the right dorsal distal metatarsal region and the tail. The skin lesion started a year ago with a crust-like lesion on the right dorsal distal metatarsal region which gradually swelled as the patient continued to lick and chew without healing. Recently, similar lesions occurred on the tail. Based on the licking history and ruling out other diseases through skin examination, acral lick dermatitis (ALD) was diagnosed. Treatment included antidepressants, preventive antibiotics, topical corticosteroid, and wearing Elizabethan collar as a physical barrier. After three weeks, the lesion on the hind limb healed entirely. However, the tail lesion recurred as it was able to lick the tail again with a type of an E-collar. This case shows that it is essential to block the contact lesion and provide medical treatment until the lesion has been completely resolved for successful management of ALD.

Key words acral lick dermatitis, dog, antidepressants, Elizabethan collar. 


\section{Introduction}

Acral lick dermatitis (ALD) is a common, frustrating canine skin disease caused by incessant licking of focal skin areas. It is characterized by incessant licking behavior resulting in well-circumscribed, raised, thickened, and ulcerative plaques. It most commonly affects lower extremities of dogs. It develops more often in male dogs of large breeds. It has a multifactorial etiology (13). ALD has various underlying causes such as tumor, infection, trauma, and orthopedic problem. It was known to be mainly caused by behavioral disorder in the past. However, in recent years, allergy has been suggested to be the most common cause (13). When the underlying cause and the perpetuating factor make the dog lick the lesion continuously, it can lead to lymphoplasmacytic dermal inflammation, acanthosis, folliculitis, furunculosis, and vertical streaking fibrosis on histopathology. The lesion then gradually develops into a firm, frustrating hair loss lesion $(12,13)$. Previously, various treatment methods such as drug, surgery, behavioral modification, laser ablation, phototherapy, radiation therapy, and acupuncture therapy have been suggested for $\operatorname{ALD}(1,2,10)$. Traditionally, antidepressants are widely used to treat obsessive compulsive disorders including $\operatorname{ALD}(5,9,10)$. However, it is likely to recur if the following three factors are not effectively controlled: underlying trigger, secondary infection, and stopping itch-lick cycle (13). Although there are several reports on treatment of a single lesion $(5,13)$, there is no report about treatment of ALD with multiple lesions in dogs. This case describes a refractory ALD with multiple lesions that should block contact with the lesion essentially along with medical treatment.

\section{Case Report}

A 7-year-old intact male Maltese dog presented with der- matologic problems. On history taking, the owner described that the skin lesion started a year ago with a crust-like injury on the right dorsal distal metatarsal region that gradually swelled and hyperpigmented as the patient continued to lick and chew without healing. The injury occasionally bled. Recently, similar lesions occurred on the tail. The patient had no history of trauma. The patient received treatment through antibiotic ointment at a local hospital, but it did not work and visited after stopping treatment for three months. The owner stated that the patient's personality was sensitive and aggressive when its paws were touched. Only one owner of the family was able to walk or wash the dog. Its exercise time was reduced to less than 3 times in a week as the owner became busy. The patient started licking feet. It even began to lick its tail about two months ago. The pruritus of the feet and tails did not indicate seasonality. There were no systemic pruritus or other skin lesions. There were no histories of allergic reactions to food or other factors (e.g., drugs, environmental factors). A $0.6 \times 0.6 \mathrm{~cm}$ plaque lesion was present on the right dorsal distal metatarsal area (Fig. 1A). The tail had excoriation lesions apparently caused by its licking and biting behavior (Fig. 2A). The patient continued to try to lick its feet and tail during the examination.

Other infectious diseases caused by parasite (e.g., Demodex, Cheyletiella), bacteria, or fungal microorganism were ruled out by skin examinations (skin tape preparation, scraping test, trichogram, smear cytology, and fungal culture). It did not show any other signs of gait or posture abnormality in orthopedic exam. It had no apparent pain reaction when its leg was palpated. Based on history taking, clinical, dermatological and orthopedic examination findings, the dog was diagnosed with ALD without secondary infections. Since there were no obvious other primary factors of ALD, it was estimated to be due to its behavior. Treatment included antidepressants (Amitriptyline, 2 mg/kg, PO bid, Dong

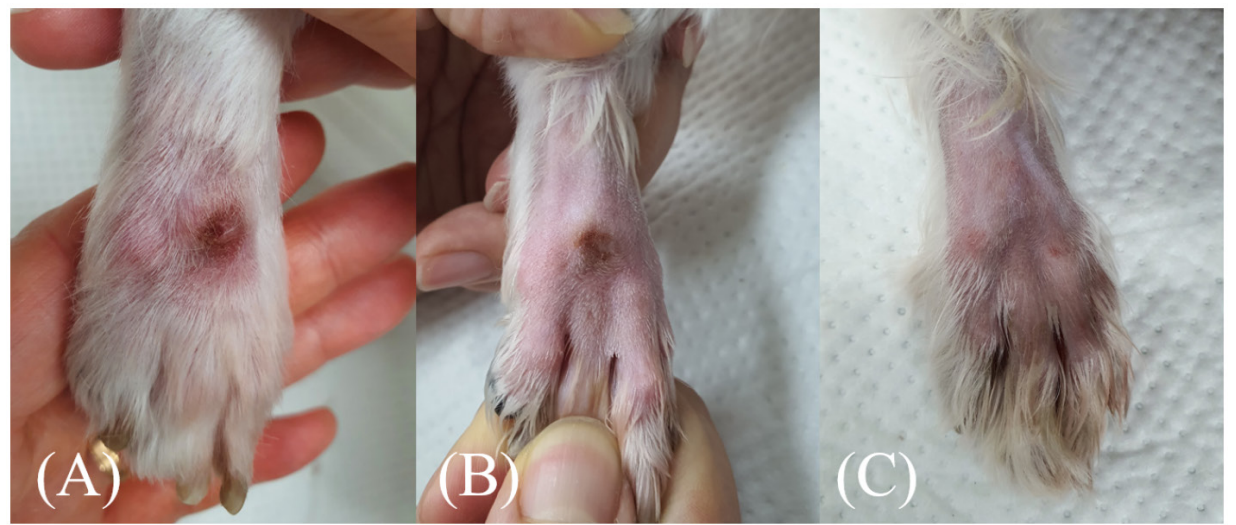

Fig. 1. Acral lick dermatitis on the right dorsal distal metatarsal region. A firm, raised, erythematous, and alopecic skin lesion was detected. (B) and (C) Pictures at one week later and three weeks later after the treatment, respectively. It had improved at three weeks after the treatment. 


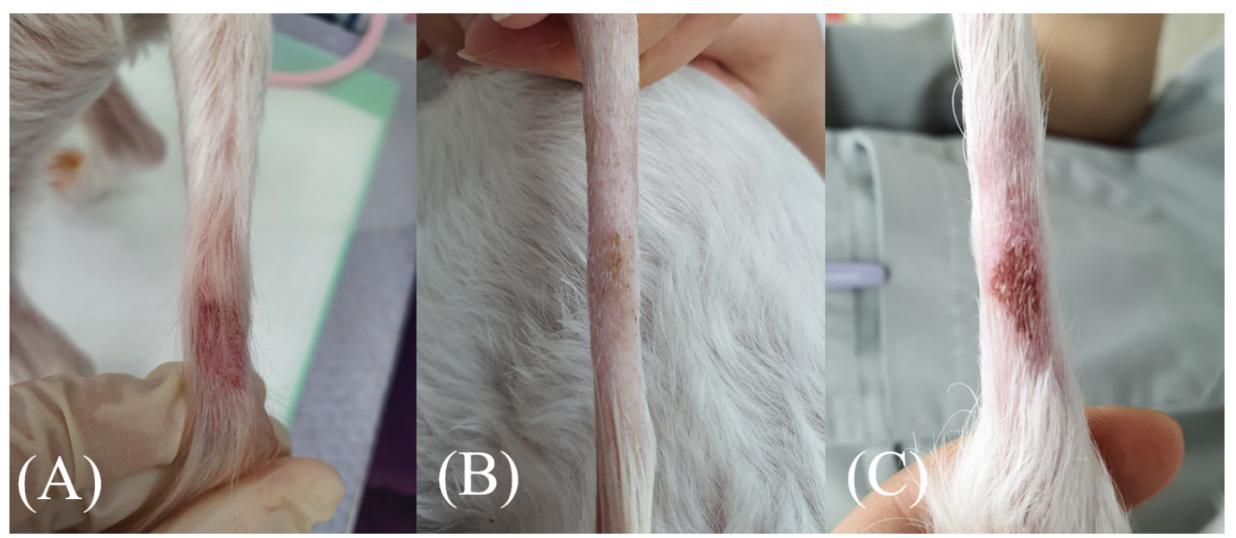

Fig. 2. Acral lick dermatitis on the tail. A firm, raised, erythematous, and alopecic skin lesion was detected. The lesion showed significant improvement one week later (B), but it relapsed after three weeks (C).

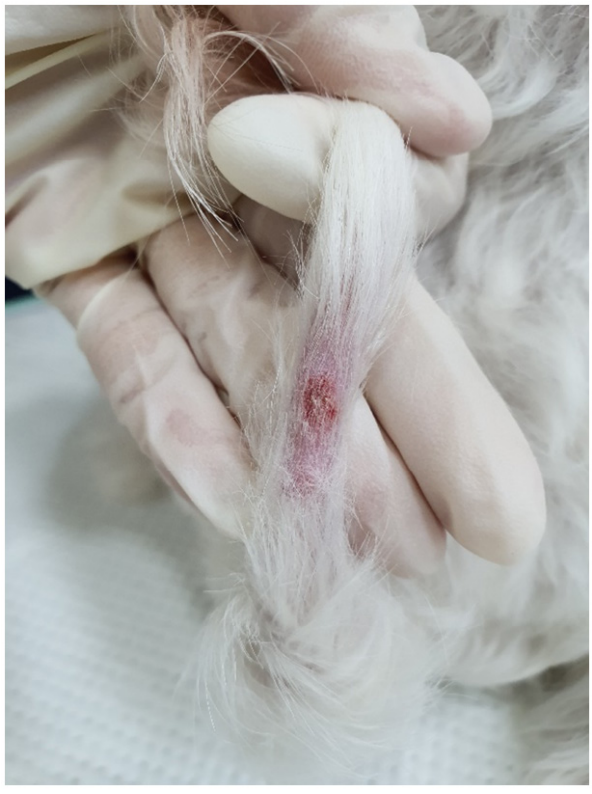

Fig. 3. The tail at six weeks after treatment. The size of the excoriation has shrunk and improved.

Hwa Pharm, Seoul, Korea), preventive antibiotics (Amoxicillin-clavulanate, $22 \mathrm{mg} / \mathrm{kg}$, PO bid, THRAGEN ETEX, Ansan, Korea), and topical corticosteroid (Namiya-G Cream, CMG Pharm, Siheung, Korea). The owner was instructed to keep her dog wearing an Elizabethan collar (E-collar) all the time and increase play time and exercise with the patient.

After a week, erythema and swelling were alleviated for both tail lesion and metatarsal lesion (Fig. 1B and Fig. 2B). On evaluation of its progress, its personality had become calmer and less aggressive than before. Two weeks later, lesion on the metatarsal area is improved. Tail lesion also showed significant improvement, although it did not completely heal. Its behavior of licking the area also disappeared (Fig. 1C). However, the lesion on the tail reoccurred (Fig. 2C) as the

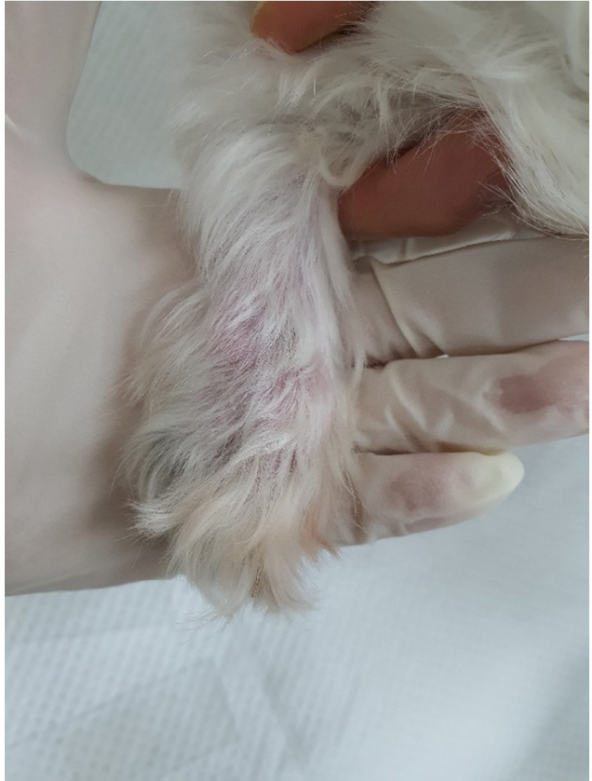

Fig. 4. The dorsal distal metatarsal region at six weeks after treatment. Hair has grown. No skin lesion has newly occurred.

owner changed his neck collar to a smaller, fluffier form of E-collar which exposes him to bite the tail again. Treatment was continued only for the tail lesion. The owner was asked to change its collar back to a large enough neck collar. Three weeks later, the tail lesion was relieved again (Fig. 3). Its metatarsal area had no new lesion (Fig. 4).

\section{Discussion}

This case shows that complete healing can be done through the use of antidepressants, preventive antibiotics, topical corticosteroids, and E-collar for three weeks in an ALD patient with multiple lesions. This case shows that protecting the lesion and blocking the licking behavior until the lesion is 
completely improved are essential.

Behavioral ALD is considered as an obsessive-compulsive disorder (OCD) in dogs (3). It has been reported that OCD dogs have alterations in serotonergic and dopaminergic neurotransmission systems (14). Serotonin regulates emotional response to stress and numerous physiological processes including sleeping, eating, aggression, and self-grooming (10). In human medicine, antidepressants and behavioral therapy are recommended to increase serotonin levels when treating skin picking disease caused by excessive picking behaviors similar to canine ALD (4). Similarly, randomized double blind experiments using selective serotonin reuptake inhibitor (SSRI) antidepressants in dogs, have demonstrated significant improvement in fluoxetine groups over placebo groups (15). Tricyclic acids (TCAs) antidepressants are also known to be effective in alleviating obsessive-compulsive disorders including ALD in dogs $(6,7,9,16)$. In one study (11), the licking behavior in a dog immediately recurred when the physical barrier was removed, corresponding to the relapse history of the present case.

Combined with previous studies, this case proved that traditional treatment using antidepressants could provide benefit to treat ALD. Blocking contact to the lesion through physical barriers is more essential in the treatment of ALD than using medication to avoid a recurrence of the lesion until all lesions are completely healed. Although antidepressants can improve the serotonergic dysfunction and suppress the compulsive behavior, the dog could concentrate on the lesion with remaining clinical symptoms (e.g., pain, erythema). Therefore, it is necessary to block the lesion until all clinical signs have completely disappeared so that the dog could forget the lesion.

Although the most commonly affected area is known as the left carpal region in ALD (13), the tail could be affected in some patients as in the present case. Unlikely limbs, it is easy to access the lesion even if wearing E-collars. Using a bandage around the lesion can also be effective. However, it is difficult to maintain a wrapping on the tail. In addition, it can interfere with blood supply and cause severe injury. In this case, it could be controlled by wearing a bigger E-collar. If a bigger E-collar is not available, alternative methods such as topical capsaicin used in human medicine should be developed in the future (8).

\section{Conclusions}

This is the first case report of partial recurrence in an ALD patient with multiple lesions. This case shows the importance of blocking contact with the lesion in ALD patients besides medical treatment.

\section{Acknowledgements}

This research was supported by the Basic Science Research Program through the National Research Foundation of Korea (NRF), funded by the Ministry of Education (NRF2020R1A2C2005364).

\section{Conflicts of Interest}

The authors have no conflicting interests.

\section{Source of Funding}

This research was supported by the Basic Science Research Program through the National Research Foundation of Korea (NRF), funded by the Ministry of Education (NRF2020R1A2C2005364).

\section{References}

1. Arza R. Using $\mathrm{CO}_{2}$ laser on acral lick granulomas. Veterinary Practice News. Apr, 2016; A special advertising section. p. 32-33.

2. Eckstein RA, Hart BL. Treatment of canine acral lick dermatitis by behavior modification using electronic stimulation. J Am Anim Hosp Assoc 1996; 32: 225-230

3. Goldberger E, Rapoport JL. Canine acral lick dermatitis: response to the antiobsessional drug clomipramine. J Am Anim Hosp Assoc 1992; 27: 179-182.

4. Grant JE, Odlaug BL, Chamberlain SR, Keuthen NJ, Lochner C, Stein DJ. Skin picking disorder. Am J Psychiatry 2012; 169: 11431149.

5. Kang MH, Lee CM, Sung HJ, Park HM. Clomipramine treatment of acral lick dermatitis in a dog. Korean J Vet Res 2013; 53: 185-187.

6. Marder AR. Psychotropic drugs and behavioral therapy. Vet Clin North Am Small Anim Pract 1991; 21: 329-342.

7. Miller WH Jr, Scott DW, Wellington JR. Nonsteroidal management of canine pruritus with amitriptyline. Cornell Vet 1992; 82: 53-57.

8. Moon JY, Lee PB, Kim YC, Lee SC, Nahm FS, Choi E. Efficacy and safety of $0.625 \%$ and $1.25 \%$ capsaicin patch in peripheral neuropathic pain: multi-center, randomized, and semi-double blind controlled study. Pain Physician 2017; 20: 27-35.

9. Overall KL, Dunham AE. Clinical features and outcome in dogs and cats with obsessive-compulsive disorder: 126 cases (19892000). J Am Vet Med Assoc 2002; 221: 1445-1452.

10. Rapoport JL, Ryland DH, Kriete M. Drug treatment of canine acral lick. An animal model of obsessive-compulsive disorder. Arch Gen Psychiatry 1992; 49: 517-521. 
11. Rehrig AN, Napolitano DA, Monsour CP. Assessment and treatment of self-injurious behavior in a dog. J Anim Behav Technol 2018; 8: 42-47.

12. Shumaker AK, Angus JC, Coyner KS, Loeffler DG, Rankin SC, Lewis TP. Microbiological and histopathological features of canine acral lick dermatitis. Vet Dermatol 2008; 19: 288-298.

13. Shumaker AK. Diagnosis and treatment of canine acral lick dermatitis. Vet Clin North Am Small Anim Pract 2019; 49: 105-123.

14. Vermeire S, Audenaert K, De Meester R, Vandermeulen E, Waelbers $T$, De Spiegeleer $B$, et al. Serotonin $2 A$ receptor, serotonin transporter and dopamine transporter alterations in dogs with compulsive behaviour as a promising model for human obsessive-compulsive disorder. Psychiatry Res 2012; 201: 78-87.

15. Wynchank D, Berk M. Fluoxetine treatment of acral lick dermatitis in dogs: a placebo-controlled randomized double blind trial. Depress Anxiety 1998; 8: 21-23.

16. Yalcin E. Comparison of clomipramine and fluoxetine treatment of dogs with tail chasing. Tierarztl Prax Ausg K Kleintiere Heimtiere 2010; 38: 295-299. 